\title{
Inflation and Transition to a Slowly Accelerating Phase from S.S.B. of Scale Invariance
}

\author{
E.I. Guendelman and O.Katz \\ Physics Department, Ben-Gurion University, Beer-Sheva 84105, Israel
}

November 19, 2018

\begin{abstract}
We consider the effects of adding a scale invariant $R^{2}$ term to the action of the scale invariant model (SIM) studied previously by one of us (E.I.G., Mod. Phys. Lett. A14, 1043 (1999)). The SIM belongs to the general class of theories, where an integration measure independent of the metric is introduced. To implement scale invariance (S.I.), a dilaton field is introduced. The integration of the equations of motion associated with the new measure gives rise to the spontaneous symmetry breaking (S.S.B) of S.I.. After S.S.B. of S.I. in the model with the $R^{2}$ term, it is found that a non trivial potential for the dilaton is generated. This potential contains two flat regions: one associated with the Planck scale and with an inflationary phase, while the other flat region is associated to a very small vacuum energy (V.E.) and is associated to the present slowly accelerated phase of the universe (S.A.PH). The smallness of the V.E. in the S.A.PH. is understood through the see saw mechanism introduced in S.I.M.
\end{abstract}

\section{Introduction}

The vacuum energy density of the universe has been invoked as a fundamental component of the energy density of the universe at least in two instances. 
First, it has been used as the source of a possible inflationary phase of the early universe ${ }^{1}$, which provides an attractive scenario for solving some of the fundamental puzzles of the standard Big Bang model, like the horizon and the flatness problems as well as providing a framework for sensible calculations of primordial density perturbations. In recent years, with the discovery of the acceleration of the present universe $^{2}$ it appears plausible that a small vacuum energy is also present even today.

These two vacuum energy densities, the one of inflation and that of the Universe now have however a totally different scale. One wonders then how cosmological evolution may naturally interpolate between such two apparently quite disconnected physical situations.

The possibility of continously connecting an inflationary phase to a slowly accelerated universe through the evolution of a single scalar field has been studied $^{3}$. In this paper we propose a theoretical framework where such scenario is realized in a natural way.

We work in the context of a two measures theory (TMT) ${ }^{4,5,6,7}$ and more specifically in the context of the scale invariant realization of such theories $5,6,7$ and will show how the stated goals can be achieved in this context.

The paper will be organized as follows: In section 2 we review the principles of the TMT and in particular the model studied in Ref. 5, which has global scale invariance. Such model gives rise, in the effective Einstein frame, to an effective potential for a dilaton field (needed to implement an interesting model with global scale invariance) which has a flat region. In section 3 we review the "cosmological see saw mechanism" introduced in Ref. 5 and which allows to obtain a small value for the vacuum energy without adjusting small numbers. In section 4 , we generalize this model by adding a curvature square or simply " $R^{2}$ term" and show that the resulting model contains now two flat regions which can be of very different scales: one must be associated to the Planck scale and is associated to an inflationary phase while the other flat region can be very small due to a "cosmological see-saw" mechanism. This very small vacuum energy may be then associated to the present accelerated universe. The existence of two flat regions for the potential is shown to be consequence of the s.s.b. of the scale symmetry. We end with a discussion and conclusions section. 


\section{The Two Measures Theories and a simple scale invariant realization}

When studying generally covariant Lagrangian formulations of gravitational theories, we usually consider the form

$$
S_{1}=\int L \sqrt{-g} d^{4} x, g=\operatorname{det} g_{\mu \nu}
$$

As it is well known, $d^{4} x$ is not a scalar but the combination $\sqrt{-g} d^{4} x$ is a scalar. Inserting $\sqrt{-g}$, which has the transformation properties of a density, produces a scalar action (1), provided $\mathrm{L}$ is a scalar.

One could use nevertheless other objects instead of $\sqrt{-g}$, provided they have the same transformation properties and achieve in this way a different generally covariant formulation.

For example, given 4-scalars $\varphi_{a}(\mathrm{a}=1,2,3,4)$, one can construct the density

$$
\Phi=\varepsilon^{\mu \nu \alpha \beta} \varepsilon_{a b c d} \partial_{\mu} \varphi_{a} \partial_{\nu} \varphi_{b} \partial_{\alpha} \varphi_{c} \partial_{\beta} \varphi_{d}
$$

and consider instead of (1) the action

$$
S_{2}=\int L \Phi d^{4} x
$$

$\mathrm{L}$ is again some scalar, which may contain the curvature (i.e. the gravitational contribution) and a matter contribution, as is standard also in (1).

In the action (3) the measure carries degrees of freedom independent of that of the metric and that of the matter fields. The most natural and successful formulation of the theory is achieved when the connection is also treated as an independent degree of freedom. This is what is usually referred to as the first order formalism.

One can notice that $\Phi$ is the total derivative of something, for example, one can write ${ }^{4,5,6,7}$

$$
\Phi=\partial_{\mu}\left(\varepsilon^{\mu \nu \alpha \beta} \varepsilon_{a b c d} \varphi_{a} \partial_{\nu} \varphi_{b} \partial_{\alpha} \varphi_{c} \partial_{\beta} \varphi_{d}\right)
$$

This means that a shift of the form

$$
L \rightarrow L+\text { constant }
$$


just adds the integral of a total divergence to the action (3) and it does not affect therefore the equations of motion of the theory. The same shift, acting on (1) produces an additional term which gives rise to a cosmological constant.

One can generalize this structure and allow both geometrical objects to enter the theory and consider

$$
S_{3}=\int L_{1} \Phi d^{4} x+\int L_{2} \sqrt{-g} d^{4} x
$$

Now instead of (5), the shift symmetry can be applied only on $L_{1}\left(L_{1} \rightarrow\right.$ $L_{1}+$ constant). Here $L_{1}$ and $L_{2}$ are $\varphi_{a}$ independent.

There is a good reason not to consider mixing of $\Phi$ and $\sqrt{-g}$, like for example using

$$
\frac{\Phi^{2}}{\sqrt{-g}}
$$

This is because (6) is invariant (up to the integral of a total divergence) under the infinite dimensional symmetry

$$
\varphi_{a} \rightarrow \varphi_{a}+f_{a}\left(L_{1}\right)
$$

where $f_{a}\left(L_{1}\right)$ is an arbitrary function of $L_{1}$ if $L_{1}$ and $L_{2}$ are $\varphi_{a}$ independent. Such symmetry (up to the integral of a total divergence) is absent if mixed terms (like (7)) are present. Therefore (6) is considered for the case when no dependence on the measure fields (MF) appears in $L_{1}$ or $L_{2}$.

We will study now the dynamics of a scalar field $\phi$ interacting with gravity as given by the following action ${ }^{5}$

$$
\begin{gathered}
S_{L}=\int L_{1} \Phi d^{4} x+\int L_{2} \sqrt{-g} d^{4} x \\
L_{1}=\frac{-1}{\kappa} R(\Gamma, g)+\frac{1}{2} g^{\mu \nu} \partial_{\mu} \phi \partial_{\nu} \phi-V(\phi) \\
L_{2}=U(\phi) \\
R(\Gamma, g)=g^{\mu \nu} R_{\mu \nu}(\Gamma), R_{\mu \nu}(\Gamma)=R_{\mu \nu \lambda}^{\lambda} \\
R_{\mu \nu \sigma}^{\lambda}(\Gamma)=\Gamma_{\mu \nu, \sigma}^{\lambda}-\Gamma_{\mu \sigma, \nu}^{\lambda}+\Gamma_{\alpha \sigma}^{\lambda} \Gamma_{\mu \nu}^{\alpha}-\Gamma_{\alpha \nu}^{\lambda} \Gamma_{\mu \sigma}^{\alpha} .
\end{gathered}
$$

The suffix $L$ in $S_{L}$ is to emphasize that here the curvature appears only linearly. 
In the variational principle $\Gamma_{\mu \nu}^{\lambda}, g_{\mu \nu}$, the measure fields scalars $\varphi_{a}$ and the "matter" - scalar field $\phi$ are all to be treated as independent variables although the variational principle may result in equations that allow us to solve some of these variables in terms of others.

There is the interesting possibility of implementing global scale invariance in such a model. Indeed, if we perform the global scale transformation $(\theta=$ constant)

$$
g_{\mu \nu} \rightarrow e^{\theta} g_{\mu \nu}
$$

then (9) is invariant provided $V(\phi)$ and $U(\phi)$ are of the form

$$
V(\phi)=f_{1} e^{\alpha \phi}, U(\phi)=f_{2} e^{2 \alpha \phi}
$$

and $\varphi_{a}$ is transformed according to

$$
\varphi_{a} \rightarrow \lambda_{a} \varphi_{a}
$$

(no sum on a) which means

$$
\Phi \rightarrow\left(\prod_{a} \lambda_{a}\right) \Phi \equiv \lambda \Phi
$$

such that

$$
\lambda=e^{\theta}
$$

and

$$
\phi \rightarrow \phi-\frac{\theta}{\alpha}
$$

We will now work out the equations of motion for arbitrary choice of $V(\phi)$ and $U(\phi)$. We study afterwards the choice (15) which allows us to obtain the results for the scale invariant case and also to see what differentiates this from the choice of arbitrary $U(\phi)$ and $V(\phi)$ in a very special way.

Let us begin by considering the equations which are obtained from the variation of the fields that appear in the measure, i.e. the $\varphi_{a}$ fields. We obtain then

$$
A_{a}^{\mu} \partial_{\mu} L_{1}=0
$$

where $A_{a}^{\mu}=\varepsilon^{\mu \nu \alpha \beta} \varepsilon_{a b c d} \partial_{\nu} \varphi_{b} \partial_{\alpha} \varphi_{c} \partial_{\beta} \varphi_{d}$. Since it is easy to check that $A_{a}^{\mu} \partial_{\mu} \varphi_{a^{\prime}}=$ $\frac{\delta a a^{\prime}}{4} \Phi$, it follows that $\operatorname{det}\left(A_{a}^{\mu}\right)=\frac{4^{-4}}{4 !} \Phi^{3} \neq 0$ if $\Phi \neq 0$. Therefore if $\Phi \neq 0$ we obtain that $\partial_{\mu} L_{1}=0$, or that

$$
L_{1}=\frac{-1}{\kappa} R(\Gamma, g)+\frac{1}{2} g^{\mu \nu} \partial_{\mu} \phi \partial_{\nu} \phi-V=M
$$


where $\mathrm{M}$ is constant.

Let us study now the equations obtained from the variation of the connections $\Gamma_{\mu \nu}^{\lambda}$. We obtain then

$-\Gamma_{\mu \nu}^{\lambda}-\Gamma_{\beta \mu}^{\alpha} g^{\beta \lambda} g_{\alpha \nu}+\delta_{\nu}^{\lambda} \Gamma_{\mu \alpha}^{\alpha}+\delta_{\mu}^{\lambda} g^{\alpha \beta} \Gamma_{\alpha \beta}^{\gamma} g_{\gamma \nu}-g_{\alpha \nu} \partial_{\mu} g^{\alpha \lambda}+\delta_{\mu}^{\lambda} g_{\alpha \nu} \partial_{\beta} g^{\alpha \beta}-\delta_{\nu}^{\lambda} \frac{\Phi_{, \mu}}{\Phi}+\delta_{\mu}^{\lambda} \frac{\Phi_{,}}{\Phi}=0$

If we define $\Sigma_{\mu \nu}^{\lambda}$ as $\Sigma_{\mu \nu}^{\lambda}=\Gamma_{\mu \nu}^{\lambda}-\left\{\begin{array}{l}\lambda \\ \mu \nu\end{array}\right\}$ where $\left\{\begin{array}{l}\lambda \\ \mu \nu\end{array}\right\}$ is the Christoffel symbol, we obtain for $\Sigma_{\mu \nu}^{\lambda}$ the equation

$$
-\sigma_{, \lambda} g_{\mu \nu}+\sigma_{, \mu} g_{\nu \lambda}-g_{\nu \alpha} \Sigma_{\lambda \mu}^{\alpha}-g_{\mu \alpha} \Sigma_{\nu \lambda}^{\alpha}+g_{\mu \nu} \Sigma_{\lambda \alpha}^{\alpha}+g_{\nu \lambda} g_{\alpha \mu} g^{\beta \gamma} \Sigma_{\beta \gamma}^{\alpha}=0
$$

where $\sigma=\ln \chi, \chi=\frac{\Phi}{\sqrt{-g}}$.

The general solution of (23) is

$$
\Sigma_{\mu \nu}^{\alpha}=\delta_{\mu}^{\alpha} \lambda,_{\nu}+\frac{1}{2}\left(\sigma_{, \mu} \delta_{\nu}^{\alpha}-\sigma_{, \beta} g_{\mu \nu} g^{\alpha \beta}\right)
$$

where $\lambda$ is an arbitrary function due to the $\lambda$ - symmetry of the curvature ${ }^{8}$ $R_{\mu \nu \alpha}^{\lambda}(\Gamma)$,

$$
\Gamma_{\mu \nu}^{\alpha} \rightarrow \Gamma_{\mu \nu}^{\alpha \alpha}=\Gamma_{\mu \nu}^{\alpha}+\delta_{\mu}^{\alpha} Z_{\nu}
$$

$\mathrm{Z}$ being any scalar (which means $\lambda \rightarrow \lambda+Z$ ).

If we choose the gauge $\lambda=\frac{\sigma}{2}$, we obtain

$$
\Sigma_{\mu \nu}^{\alpha}(\sigma)=\frac{1}{2}\left(\delta_{\mu}^{\alpha} \sigma_{, \nu}+\delta_{\nu}^{\alpha} \sigma_{, \mu}-\sigma_{, \beta} g_{\mu \nu} g^{\alpha \beta}\right) .
$$

Considering now the variation with respect to $g^{\mu \nu}$, we obtain

$$
\Phi\left(\frac{-1}{\kappa} R_{\mu \nu}(\Gamma)+\frac{1}{2} \phi,_{\mu} \phi,_{\nu}\right)-\frac{1}{2} \sqrt{-g} U(\phi) g_{\mu \nu}=0
$$

solving for $R=g^{\mu \nu} R_{\mu \nu}(\Gamma)$ from (27) and introducing in (21), we obtain

$$
M+V(\phi)-\frac{2 U(\phi)}{\chi}=0
$$

a constraint that allows us to solve for $\chi$,

$$
\chi=\frac{2 U(\phi)}{M+V(\phi)} .
$$


To get the physical content of the theory, it is convenient to go to the Einstein conformal frame where

$$
\bar{g}_{\mu \nu}=\chi g_{\mu \nu}
$$

and $\chi$ given by $(29 \mathrm{~b})$. In terms of $\bar{g}_{\mu \nu}$ the non Riemannian contribution $\Sigma_{\mu \nu}^{\alpha}$ dissappears from the equations. This is because the connection can be written as the Christoffel symbol of the metric $\bar{g}_{\mu \nu}$. In terms of $\bar{g}_{\mu \nu}$ the equations of motion for the metric can be written then in the Einstein form (we define $\bar{R}_{\mu \nu}\left(\bar{g}_{\alpha \beta}\right)=$ usual Ricci tensor in terms of the bar metric $=R_{\mu \nu}$ $\left.\bar{R}=\bar{g}^{\mu \nu} \bar{R}_{\mu \nu}\right)$

$$
\bar{R}_{\mu \nu}\left(\bar{g}_{\alpha \beta}\right)-\frac{1}{2} \bar{g}_{\mu \nu} \bar{R}\left(\bar{g}_{\alpha \beta}\right)=\frac{\kappa}{2} T_{\mu \nu}^{e f f}(\phi)
$$

where

$$
T_{\mu \nu}^{e f f}(\phi)=\phi_{, \mu} \phi_{, \nu}-\frac{1}{2} \bar{g}_{\mu \nu} \phi_{, \alpha} \phi_{, \beta} \bar{g}^{\alpha \beta}+\bar{g}_{\mu \nu} V_{e f f}(\phi)
$$

and

$$
V_{e f f}(\phi)=\frac{1}{4 U(\phi)}(V+M)^{2} .
$$

In terms of the metric $\bar{g}^{\alpha \beta}$, the equation of motion of the Scalar field $\phi$ takes the standard General - Relativity form

$$
\frac{1}{\sqrt{-\bar{g}}} \partial_{\mu}\left(\bar{g}^{\mu \nu} \sqrt{-\bar{g}} \partial_{\nu} \phi\right)+V_{e f f}^{\prime}(\phi)=0
$$

Notice that if $V+M=0, V_{e f f}=0$ and $V_{e f f}^{\prime}=0$ also, provided $V^{\prime}$ is finite and $U \neq 0$ there. This means the zero cosmological constant state is achieved without any sort of fine tuning. That is, independently of whether we add to $V$ a constant piece, or whether we change the value of $M$, as long as there is still a point where $V+M=0$, then still $V_{\text {eff }}=0$ and $V_{\text {eff }}^{\prime}=0$ ( still provided $V^{\prime}$ is finite and $U \neq 0$ there). This is the basic feature that characterizes the TMT and allows it to solve the 'old' cosmological constant problem ${ }^{4}$ at least at the classical level.

In what follows we will study (33) for the special case of global scale invariance, which as we will see displays additional very special features which makes it attractive in the context of cosmology. 
Notice that in terms of the variables $\phi, \bar{g}_{\mu \nu}$, the "scale" transformation becomes only a shift in the scalar field $\phi$, since $\bar{g}_{\mu \nu}$ is invariant (since $\chi \rightarrow$ $\lambda^{-1} \chi$ and $\left.g_{\mu \nu} \rightarrow \lambda g_{\mu \nu}\right)$

$$
\bar{g}_{\mu \nu} \rightarrow \bar{g}_{\mu \nu}, \phi \rightarrow \phi-\frac{\theta}{\alpha} .
$$

If $V(\phi)=f_{1} e^{\alpha \phi}$ and $U(\phi)=f_{2} e^{2 \alpha \phi}$ as required by scale invariance (14), (16), (17), (18), (19), we obtain from (33)

$$
V_{e f f}=\frac{1}{4 f_{2}}\left(f_{1}+M e^{-\alpha \phi}\right)^{2}
$$

Since we can always perform the transformation $\phi \rightarrow-\phi$ we can choose by convention $\alpha>0$. We then see that as $\phi \rightarrow \infty, V_{\text {eff }} \rightarrow \frac{f_{1}^{2}}{4 f_{2}}=$ const. providing an infinite flat region as depicted in Fig. 1. Also a minimum is achieved at zero cosmological constant for the case $\frac{f_{1}}{M}<0$ at the point

$$
\phi_{\min }=\frac{-1}{\alpha} \ln \left|\frac{f_{1}}{M}\right| .
$$

Finally, the second derivative of the potential $V_{\text {eff }}$ at the minimum is

$$
V_{e f f}^{\prime \prime}=\frac{\alpha^{2}}{2 f_{2}}\left|f_{1}\right|^{2}>0
$$

if $f_{2}>0$, there are many interesting issues that one can raise here. The first one is of course the fact that a realistic scalar field potential, with massive exitations when considering the true vacuum state, is achieved in a way consistent with the idea of scale invariance.

A peculiar feature of the potential (36), is that the absolute value of the constant $M$, does not affect the physics of the problem, only the sign will have an effect. This is because if we perform a shift

$$
\phi \rightarrow \phi+\Delta
$$

in the potential (36), this is equivalent to the change in the integration constant M

$$
M \rightarrow M e^{-\alpha \Delta} .
$$


We see therefore that if we change $M$ in any way, without changing the sign of M, the only effect this has is to shift the whole potential. The physics of the potential remains unchanged, however. This is reminiscent of the dilatation invariance of the theory, which involves only a shift in $\phi$ if $\bar{g}_{\mu \nu}$ is used (see eq. (35)).

This is very different from the situation for two generic functions $U(\phi)$ and $V(\phi)$ in (33). There, $\mathrm{M}$ appears in $V_{\text {eff }}$ as a true new parameter that generically changes the shape of the potential $V_{\text {eff }}$, i.e. it is impossible then to compensate the effect of $M$ with just a shift. For example $M$ will appear in the value of the second derivative of the potential at the minimum, unlike what we see in eq. (38), where we see that $V_{e f f}^{\prime \prime}(\min )$ is M independent.

In conclusion, the scale invariance of the original theory is responsible for the non appearance (in the physics) of a certain scale, that associated to M. However, masses do appear, since the coupling to two different measures of $L_{1}$ and $L_{2}$ allow us to introduce two independent couplings $f_{1}$ and $f_{2}$, a situation which is unlike the standard formulation of globally scale invariant theories, where usually no stable vacuum state exists.

Notice that we have not considered all possible terms consistent with global scale invariance. Additional terms in $L_{2}$ of the form $e^{\alpha \phi} R$ and $e^{\alpha \phi} g^{\mu \nu} \partial_{\mu} \phi \partial_{\nu} \phi$ are indeed consistent with the global scale invariance (14), (16), (17), (18), (19) but they give rise to a much more complicated theory, which has been studied in a separate publication ${ }^{7}$. There, when the theory is studied in the presence of fermions, the equation that determines the ratio between the two measures becomes non linear and the multiple solutions of the constraint equation provide an oportunity for explaining the family structure of fermions ${ }^{7}$ and other interesting cosmological questions.

We can compare the appearance of the potential $V_{\text {eff }}(\phi)$, which has privileged some point depending on $\mathrm{M}$ (for example the minimum of the potential will have to be at some specific point), although the theory has the "translation invariance" (35), to the physics of solitons.

In fact, this very much resembles the appearance of solitons in a spacetranslation invariant theory: The soliton solution has to be centered at some point, which of course is not determined by the theory. The soliton of course breaks the space translation invariance spontaneously, just as the existence of the non trivial potential $V_{\text {eff }}(\phi)$ breaks here spontaneously the translations in $\phi$ space, since $V_{\text {eff }}(\phi)$ is not a constant.

The constant of integration $M$ plays a very important role indeed: any 
non vanishing value for this constant implements, already at the classical level S.S.B. of scale invariance.

\section{The Cosmological See Saw Mechanism}

Notice the existence for $\phi \rightarrow \infty$, of a flat region for $V_{\text {eff }}(\phi)$ can be nicely described as a region where the symmetry under translations (35) is restored.

One should point out that the model discussed here gives a potential with two physically relevant parameters : $\frac{f_{1}^{2}}{4 f_{2}}$, which represents the value of $V_{\text {eff }}$ as $\phi \rightarrow \infty$, i.e. the strength of the false vacuum at the flat region and $\frac{\alpha^{2} f_{1}^{2}}{2 f_{2}}$ , representing the mass of the excitations around the true vacuum with zero cosmological constant (achieved here without fine tuning).

One can consider this model as suitable for the present universe and the almost constant vacuum energy for for large values of the scalar field $f_{1}^{2} / 4 f_{2}$ can be the source of the acceleration now.

Notice that a small value of $\frac{f_{1}^{2}}{f_{2}}$ can be achieved if we let $f_{2}>>f_{1}$. In this case $\frac{f_{1}^{2}}{f_{2}}<<f_{1}$, i.e. a very small scale for the the vacuum energy can be achieved by a sort of see-saw mechanism that resembles the neutrino see saw effect ( Ref. 9). If the scale of $f_{1}$ is the electroweak scale and the scale of $f_{2}$ is the Planck scale, then naturally we are led to a small vacuum energy for the present universe of the order of $M_{E W}^{8} / M_{P l}^{4}$. This is also of the correct order of magnitude. It is exactly what is needed in the new approach to cosmic coincidences discussed by Arkani-Hamed et. al. ${ }^{10}$.

One should recall that a flat region of a potential is also a desirable feature in the case of inflationary scenarios. So far we have obtained a potential with a single flat region, so it could be used for either either providing a source of inflation or for providing a very small vacuum energy for the present universe but not both. In the next section we will see how the addition of a curvature square term remedies this situation. 


\section{The scale invariant TMT with an addi- tional $R^{2}$ term}

The simple model of section 2 , which contains a single flat region can do a good job at describing either inflation or a slowly accelerated universe. But what about explaining both, including a possible transition between these two epochs?.

A simple generalization of the action $S_{L}$ will do this job. What one needs to do is simply consider the addition of a scale invariant term of the form

$$
S_{R^{2}}=\epsilon \int\left(g^{\mu \nu} R_{\mu \nu}(\Gamma)\right)^{2} \sqrt{-g} d^{4} x
$$

The total action being then $S=S_{L}+S_{R^{2}}$. In the first order formalism $S_{R^{2}}$ is not only globally scale invariant but also locally scale invariant, that is conformally invariant (recall that in the first order formalism the connection is an independent degree of freedom and it does not transform under a conformal transformation of the metric).

Let us see what the equations of motion tell us, now with the addition of $S_{R^{2}}$ to the action. First of all, since the addition has been only to the part of the action that couples to $\sqrt{-g}$, the equations of motion derived from the variation of the measure fields remains unchanged. That is eq. (21) remains valid.

The variation of the action with respect to $g^{\mu \nu}$ gives now

$$
R_{\mu \nu}(\Gamma)\left(\frac{-\Phi}{\kappa}+2 \epsilon R \sqrt{-g}\right)+\Phi \frac{1}{2} \phi,{ }_{\mu} \phi,_{\nu}-\frac{1}{2}\left(\epsilon R^{2}+U(\phi)\right) \sqrt{-g} g_{\mu \nu}=0
$$

It is interesting to notice that if we contract this equation with $g^{\mu \nu}$, the $\epsilon$ terms do not contribute. This means that the same value for the scalar curvature $R$ is obtained as in section 2, if we express our result in terms of $\phi$, its derivatives and $g^{\mu \nu}$. Solving the scalar curvature from this and inserting in the other $\epsilon$ - independent equation $L_{1}=M$ we get still the same solution for the ratio of the measures which was found in section 2, i.e. $\chi=\frac{\Phi}{\sqrt{-g}}=\frac{2 U(\phi)}{M+V(\phi)}$.

In the presence of the $\epsilon R^{2}$ term in the action, eq. (22) gets modified so that instead of $\Phi, \Omega=\Phi-2 \epsilon R \sqrt{-g}$ appears. This in turn implies that 
eq. (23) mantains its form but where $\sigma$ is replaced by $\omega=\ln \left(\frac{\Omega}{\sqrt{-g}}\right)=$ $\ln (\chi-2 \kappa \epsilon R)$, where once again, $\chi=\frac{\Phi}{\sqrt{-g}}=\frac{2 U(\phi)}{M+V(\phi)}$.

Following then the same steps as in section 2, we can then verify that the connection is the Christoffel symbol of the metric $\bar{g}_{\mu \nu}$ given by

$$
\bar{g}_{\mu \nu}=\left(\frac{\Omega}{\sqrt{-g}}\right) g_{\mu \nu}=(\chi-2 \kappa \epsilon R) g_{\mu \nu}
$$

$\bar{g}_{\mu \nu}$ defines now the "Einstein frame". Equations (42) can now be expressed in the "Einstein form"

$$
\bar{R}_{\mu \nu}-\frac{1}{2} \bar{g}_{\mu \nu} \bar{R}=\frac{\kappa}{2} T_{\mu \nu}^{e f f}
$$

where

$$
T_{\mu \nu}^{e f f}=\frac{\chi}{\chi-2 \kappa \epsilon R}\left(\phi_{, \mu} \phi_{, \nu}-\frac{1}{2} \bar{g}_{\mu \nu} \phi_{, \alpha} \phi_{, \beta} \bar{g}^{\alpha \beta}\right)+\bar{g}_{\mu \nu} V_{e f f}
$$

where

$$
V_{e f f}=\frac{\epsilon R^{2}+U}{(\chi-2 \kappa \epsilon R)^{2}}
$$

Here it is satisfied that $\frac{-1}{\kappa} R(\Gamma, g)+\frac{1}{2} g^{\mu \nu} \partial_{\mu} \phi \partial_{\nu} \phi-V=M$, equation that expressed in terms of $\bar{g}^{\alpha \beta}$ becomes

$\frac{-1}{\kappa} R(\Gamma, g)+(\chi-2 \kappa \epsilon R) \frac{1}{2} \bar{g}^{\mu \nu} \partial_{\mu} \phi \partial_{\nu} \phi-V=M$. This allows us to solve for $R$ and we get,

$$
R=\frac{-\kappa(V+M)+\frac{\kappa}{2} \bar{g}^{\mu \nu} \partial_{\mu} \phi \partial_{\nu} \phi \chi}{1+\kappa^{2} \epsilon \bar{g}^{\mu \nu} \partial_{\mu} \phi \partial_{\nu} \phi}
$$

Notice that if we express $R$ in terms of $\phi$, its derivatives and $g^{\mu \nu}$, the result is the same as in section 2 , this is not true anymore once we express $R$ in terms of $\phi$, its derivatives and $\bar{g}^{\mu \nu}$.

In any case, once we insert (47) into (46), we see that the effective potential (46) depends on the derivatives of the scalar field. It acts as a normal scalar field potential under the conditions of slow rolling or low gradients and in the case the scalar field is near the region $M+V(\phi)=0$.

Notice that since $\chi=\frac{2 U(\phi)}{M+V(\phi)}$, then if $M+V(\phi)=0$, then, as in section 2, we obtain that $V_{\text {eff }}=V_{\text {eff }}^{\prime}=0$ at that point without fine tuning (here 
by $V_{e f f}^{\prime}$ we mean the derivative of $V_{\text {eff }}$ with respect to the scalar field $\phi$, as usual).

In the case of the scale invariant case, where $V$ and $U$ are given by equation (15), it is interesting to study the shape of $V_{\text {eff }}$ as a function of $\phi$ in the case of a constant $\phi$, in which case $V_{\text {eff }}$ can be regarded as a real scalar field potential. Then from (47) we get $R=-\kappa(V+M)$, which inserted in (46) gives,

$$
V_{e f f}=\frac{\left(f_{1} e^{\alpha \phi}+M\right)^{2}}{4\left(\epsilon \kappa^{2}\left(f_{1} e^{\alpha \phi}+M\right)^{2}+f_{2} e^{2 \alpha \phi}\right)}
$$

a typical shape for $V_{\text {eff }}$ for $\epsilon=0$ and $\frac{f_{1}}{M}<0$ is depicted in Fig. 1 . This corresponds to the potential of section 2. In contrast, for $\epsilon>0$, the corresponding potential shows two flat regions as it is explicitly shown in Fig. 2 (for $\frac{f_{1}}{M}<0$ ) and in Fig. 3 (for $\frac{f_{1}}{M}>0$ ).

The limiting values of $V_{\text {eff }}$ are:

First, for asymptotically large positive values, ie. as $\alpha \phi \rightarrow \infty$, we have $V_{e f f} \rightarrow \frac{f_{1}^{2}}{4\left(\epsilon \kappa^{2} f_{1}^{2}+f_{2}\right)}$ and under the see saw conditions discussed in section 3 , we see that the correction in the denominator, i.e. $\epsilon \kappa^{2} f_{1}^{2}$, is very small compared to the other term in the denominator, $f_{2}$. This is easy to see, since $\kappa^{2}=\frac{1}{M_{P l}^{4}}$ and we took in section 3 that $f_{1}=M_{E W}^{4}<<f_{2}=M_{P l}^{4}$, so that indeed $\epsilon \kappa^{2} f_{1}^{2}<<f_{2}$ and the see saw effect discussed in section 3 is then unchanged by the presence of an $R^{2}$ term.

Second, for asymptotically large but negative values of the scalar field, that is as $\alpha \phi \rightarrow-\infty$, we have: $V_{\text {eff }} \rightarrow \frac{1}{4 \epsilon \kappa^{2}}$, which if $\epsilon$ is a number of order one, it means we have an energy density determined by the Planck scale. This region is suitable for inflation.

In these two asymptotic regions $(\alpha \phi \rightarrow \infty$ and $\alpha \phi \rightarrow-\infty)$ an examination of the scalar field equation reveals that a constant scalar field configuration is a solution of the equations, as is of course expected from the flatness of the effective potential in these regions.

Notice that in all the above discussion it is fundamental that $M \neq 0$. If $M=0$ the potential becomes just a flat one, $V_{e f f}=\frac{f_{1}^{2}}{4\left(\epsilon \kappa^{2} f_{1}^{2}+f_{2}\right)}$ everywhere (not only at high values of $\alpha \phi$ ). The other flat region associated to the Planck scale and inflation is lost. That is the spontaneous generation of the constant of integration $M$ plays a fundamental role in the construction of the scenario where the universe starts in an inflationary phase and ends up in a slowly 
accelerated phase. As we discussed in section $2, M \neq 0$ implies the we are considering a situation with S.S.B. of scale invariance.

\section{Discussion and Conclusions}

We have seen how the addition of an $R^{2}$ term gives rise to a situation where it is possible to interpolate between an inflationary phase and a slowly accelerated phase. This is evident after reformulating the theory in the "Einstein frame" and considering the situation of slow rolling (i.e. spacetime gradients of the scalar field are small).

We find that this is accomplished because the effective potential has now two asymptotically flat regions instead of only one found in Ref. 5. The $\epsilon R^{2}$ term is shown to have the effect of cutting off the part of the effective potential where it went to infinity. Now instead, this region becomes flat. The hight of this flat region is determined by the Planck scale.

In some previous papers 11,12 "quintessential inflation" in the context of TMT was studied. In Ref. 11, starting from eq. (33) some special choices for $V$ and $U$ were shown to give a "quintesential form" for the potential which could allow an inflationary phase and then approach to a slowly accelerated phase also. These choices break scale invariance and as a result there is a great degree of arbitrariness on the way the resulting potential turns out. These problems are not present in the formulation presented here. In Ref. 12 , in the context of a scale invariant theory, a potential containing a flat

region followed by a decaying to zero piece was obtained. This could also in principle provide a scenario for inflation followed by a slowly accelerated universe. The problem with the model of Ref. 12 is its complexity, for example four index field strengths have to be introduced, in addition to the the field content of the model explained here. On the other hand, only one measure $(\Phi$ and not $\sqrt{-g}$ ) needs to be used.

The model we discuss here belongs still to the general class of models refered to as 'quintessence' (see Refs.3 and 13 for some papers on this subject), if we mean by this that the energy density of the universe is dictated by the dynamics of a scalar field (some people would add to the definition the condition that the scalar potential goes to zero as the univese evolve, in this case, this depends on our prejudices concerning the history of the universe, i.e. if ends up in the absolute minimum of fig. 2 in the very far future of the 
history of the universe, for example). Such quintessence scenarios, without the additional ingredient of an early inflation were discussed in Refs. 5,6,7 as well in a totally scale invariant framework. The addition of the $\epsilon R^{2}$ allows a succesfull generalization that allows inflation to be incorporated as we have seen.

The addition of the $\epsilon R^{2}$ term does not affect the cosmological see saw effect for the other asymptotically flat region, that is the see saw effect is stable under the addition of the $\epsilon R^{2}$ terms. The $\epsilon$ contributions are subdominant. Therefore we get in the new model two flat regions: one associated to a high (Planck) scale and the other which is associated to a very small vacuum energy density. This is indeed a promising framework for describing the full evolution of the Universe starting from inflation and ending up in the slowly accelerated phase today.

In order for the whole idea to work we need to take $M \neq 0$, so the effective potential has indeed two flat regions. $M \neq 0$ means that we are considering a situation with S.S.B. of scale symmetry. We can therefore say that the S.S.B. of scale symmetry is the source of the inflationary phase and that of a smooth transition to a slowly accelerated phase today.

As we have seen, in order to get two flat regions instead of just one, it is needed that $M \neq 0$, which corresponds to considering a situation where S.S.B. of scale invariance has taken place.

It should be pointed out also that the $R^{2}$ theory studied here, in the context of an action that contains a measure $\Phi$ independent of the metric and with the use of the first order formalism (the connections are considered as independent degrees of freedom in the action principle), leads to equations of motion that are only second order, i.e. only second derivatives of the metric and the matter fields appear (although higher powers of the derivatives of the scalar field do appear). This after the new measure $\Phi$ and the connections are solved (through the equations of motion) in terms of the metric and matter fields and after we express our results in the Einstein frame.

This is in contrast to the usual $R^{2}$ theories in the second order formalism and with standard measure everywhere in the action ${ }^{14}$. In this case, these theories lead to fourth order equatios for the metric field. In Ref. 14 it was shown that the fourth order structure of the equations can be reformulated as a system of second order equations which contain however one additional degree of freedom, a scalar field. In the case of the usual $R^{2}$ theories this scalar field contains a potential with a flat region, as that of Fig. 1, with 
an energy scale which is of the Planck scale. A second flat region is missing however so that the model can describe inflation but not inflation plus a slowly accelerated phase. For studies of inflation in the usual $R^{2}$ theories in the second order formalism, see Ref. 15.

\section{Acknowledgements}

We would like to thank E.Bogomolny and E.Nissimov for much help in the preparation of this manuscript and to R. Brustein, V. Burdyuzha, A. Davidson A. Kaganovich and E.Nissimov for conversations on the subjects discussed here. 


\section{References}

[1] For a non technical review and a good collection of further references on different aspects of inflation see A. Guth, "The Inflationary Universe", Vintage, Random House (1998). For a more technical review see E.W. Kolb and M.S. Turner, "The Early Universe", Addison Wesley (1990). The pioneer papers on the subject were A.H. Guth, Phys. Rev. D23, 347 (1981); A.D. Linde, Phys. Lett, 108B, (1982) 389; A. Albrecht and P.J. Steinhardt, Phys. Rev. Lett, 48, (1982) 1220.

[2] For reviews of this subject see for example M.S. Turner in the third Stromle Symposium: "The Galactic Halo", ASP Conference Series, Vol 666, 1999, (eds) B.K. Gibson, T.S. Axelrod and M.E. Putman and N. Bahcall, J.P. Ostriker, S.J. Perlmutter and P.J. Steinhardt, Science 284, 1481 (1999).

[3] P.J.E. Peebles and A.Vilenkin, Phys. Rev. D59: 063505 (1999).

[4] For a review of issues concerning the Two Measures Theory on subjects not related to scale invariance, see E.I. Guendelman and A.B. Kaganovich, Phys. Rev., D60: 065004 (1999); some previous works on this subject are: E.I. Guendelman and A.B. Kaganovich, Phys. Rev., D53, (1996) 7020; E.I. Guendelman and A.B. Kaganovich, Proceedings of the third Alexander Friedmann International Seminar on Gravitation and Cosmology, ed. by Yu. N. Gneding, A.A. Grib and V.M. Mostepanenko (Friedmann laboratory Publishing, st. Petersburg, 1995); E.I. Guendelman and A.B. Kaganovich, Phys. Rev., D55, (1997) 5970; E.I. Guendelman and A.B. Kaganovich, Mod. Phys. Lett, A12, (1997) 2421; E.I. Guendelman and A.B. Kaganovich, Phys. Rev., D56, (1997) 3548; E.I. Guendelman and A.B. Kaganovich, Hadronic Journal, 21, (1998) 19; E.I. Guendelman and A.B. Kaganovich, Mod. Phys. Lett., A13, (1998) 1583; F. Gronwald, U. Muench and F.W. Hehl, Hadronic Journal, 21, (1998) 3; E.I. Guendelman and A.B. Kaganovich, Phys. Rev., D57, (1998) 7200; E.I. Guendelman and A.B. Kaganovich, "Gravity Cosmology and Particle Field Dynamics without the Cosmological Constant Problem", in the Proceedings of the sixth International Symposium on Particle, Strings and Cosmolgy, PASCO5-98, World Scientific, Singapore, 1999; E.I. Guendelman and A.B. Kaganovich, "Field 
Theory Models without the Cosmological Constant problem", Plenary talk (given by E.I. Guendelman) on the fourth Alexander Friedmann International Seminar on Gravitation and Cosmology, Gr-qc/9809052; E.I. Guendelman, Int. Journ. of Mod. Phys. A14: 3497 (1999).

[5] E.I. Guendelman, Mod. Phys. Lett. A14: 1043 (1999).

[6] E.I. Guendelman, Mod. Phys. Lett. A14: 1397 (1999); E.I. Guendelman, Class. and Quantum. Grav. 17: 361 (2000); E.I.Guendelman, "Scale invariance, mass and cosmology" gr-qc/9901067; E.I.Guendelman, "Scale invariance and the present vacuum energy of the universe", contribution to the 35-th Rencontres de Moriond, gr-qc/0004011; E.I.Guendelman, "Measure fields, the cosmological constant and scale invariance" contribution to the 30-th ICHEP conference, hep-th/0008122.

[7] E.I. Guendelman and A.B.Kaganovich, hep-th/0106152; E.I. Guendelman and A.B.Kaganovich, Int. Journ. Mod. Phys. A17: 417 (2002) ; E.I. Guendelman and A.B.Kaganovich, Mod. Phys. Lett. A17: 1227 (2002).

[8] A. Einstein, "The Meaning of Relativity", MJF books, NY (1956), see appendix II.

[9] M. Gell-Mann, P. Ramond and R. Slansky, in Supergravity, edited by D. Friedman (North Holland, Amsterdam, 1979)p. 315; T. Yanagida in Proceedings of the Workshop on "Unified Theory and Baryon Number in the Universe", edited by O. Sawada and A. Sugamoto (KEK, Tsukuba, Japan, 1979); R. Mohapatra and G. Senjanovic, Phys. Rev. Lett., 44, (1980), 912 and Phys. Rev., D23, (1981) 165; A. Davidson and K.C. Wali, Phys. Rev. Lett., 59 (1987) 393.

[10] N.Arkani-Hamed, L.J.Hall, C.Kolda and H.Murayama, Phys. Rev. Lett. 85: 4434 (2000); E.I.Guendelman, "A new measure, scale invariance and see saw cosmology", contribution to PASCOS 2001; A.D.Chernin, astro-ph/0101532. Other related works are: S. Huber and Q. Shafi, hep-ph/0207732; S.Kashru, J.Kumar and E. Silverstein, Phys. Rev. D59 (1999): 106004; S.Kashru, M. Shulz and E. Silverstein, Phys. Rev. D62(200): 045021; P.H.Frampton, hep-th/ 0002053; T.Banks and M.Dine, hep-th/0106276; S.M.Barr and D.Seckel, Phys. Rev. D64 (2001) 123513. 
[11] A.B. Kaganovich, Phys. Rev. D63: 025022 (2001).

[12] E.I.Guendelman, Foundations of Physics 31: 1019 (2001). See section entitled "Scale invariant quintessential models".

[13] Some references on this subject are: C.Wetterich, Nucl. Phys. B302, 668 (1988); B.Ratra and P.J.E.Peebles, Phys. Rev. D 37, 3406 (1988); P.J.E.Peebles and B.Ratra, Astrophys. J. 325, L17 (1988); R.Caldwell, R.Dave and P.Steinhardt, Phys. Rev. Lett. 80, 1582 (1998); N.Weiss, Phys. Lett. B197, 42 (1987); Y.Fujii and T.Nishioka, Phys. Rev. D 42, 361 (1990); M.S.Turner and M.White, Phys. Rev. D56R, 4439 (1997); E.Copeland, A.Liddle and D.Wands, Phys. Rev. D 57, 4686 (1998); C.T.Hills, D.N.Schramm and J.N.Fry, Comments Nucl.Part. Phys.19, 25 (1989); J.Frieman, C.Hill and R.Watkins, Phys. Rev. D46, 1226 (1992); J.Frieman, C.Hill, A.Stebbins and I.Waga, Phys. Rev. Lett. 75, 2077 (1995); C.Wetterich, Nucl. Phys. B302, 645 (1988); Astron. Astrophys. 301, 321(1995); P.Ferreira and M.Joyce, Phys. Rev. Lett. 79, 4740 (1997); Phys. Rev. D 58, 023503 (1998); I.Zlavtev, L.Wang and P.Steinhardt, Phys. Rev. Lett. 82, 896 (1999); P.Steinhardt, L.Wang and I.Zlatev, Phys. Rev. D 59, 123504 (1999).

[14] See for example J.D.Barrow and S. Cotsakis, Phys. Lett. B214: 515 (1988); J.D.Barrow and A.C.Otterwill, J.Phys. A: Math. Gen.16, 2757 (1983); P.W.Higgs, Nuovo Cimento 11 (1959) 816 and B.Witt, Phys Lett B145 (1984) 176. If and arbitrary function of the scalar curvature appears see G.Mangano, M.Ferraris and M.Francaviglia, Gen. Rel. Grav. 19 (1987) 465 and M.Ferraris, M. Francaviglia and G. Mangano Class. Quantum Grav. 5 (1988) L95.

[15] M.B.Mijic, M.M.Morris and W-M Suen, Phys. Rev. D34, 2934 (1986). The case when there is an additional scalar in the theory has been studied by C.Contreras, R.Herrera and S. del Campo, Class. and Quantum Grav. 12 , 1937 (1995). The first inflationary model from a model with higher terms in the curvature was proposed in A.A. Starobinsky, Phys. Lett. 91B, 99 (1980). 


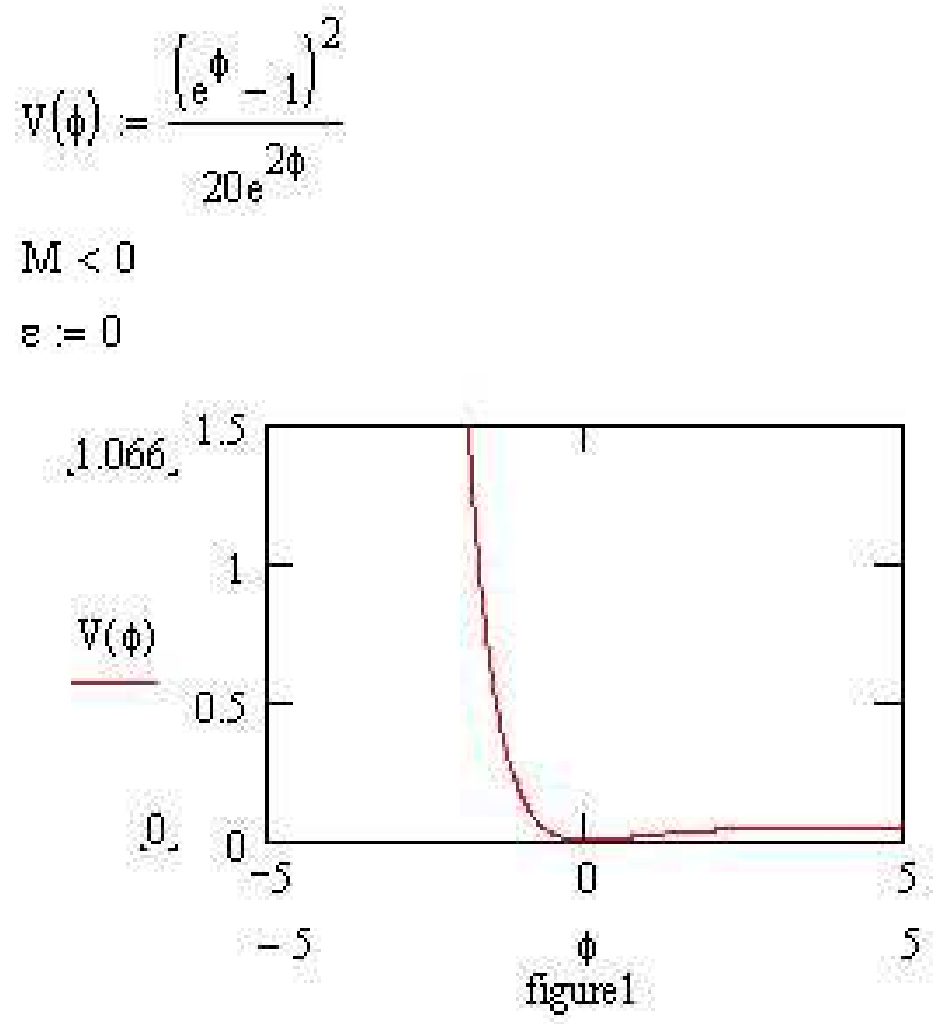




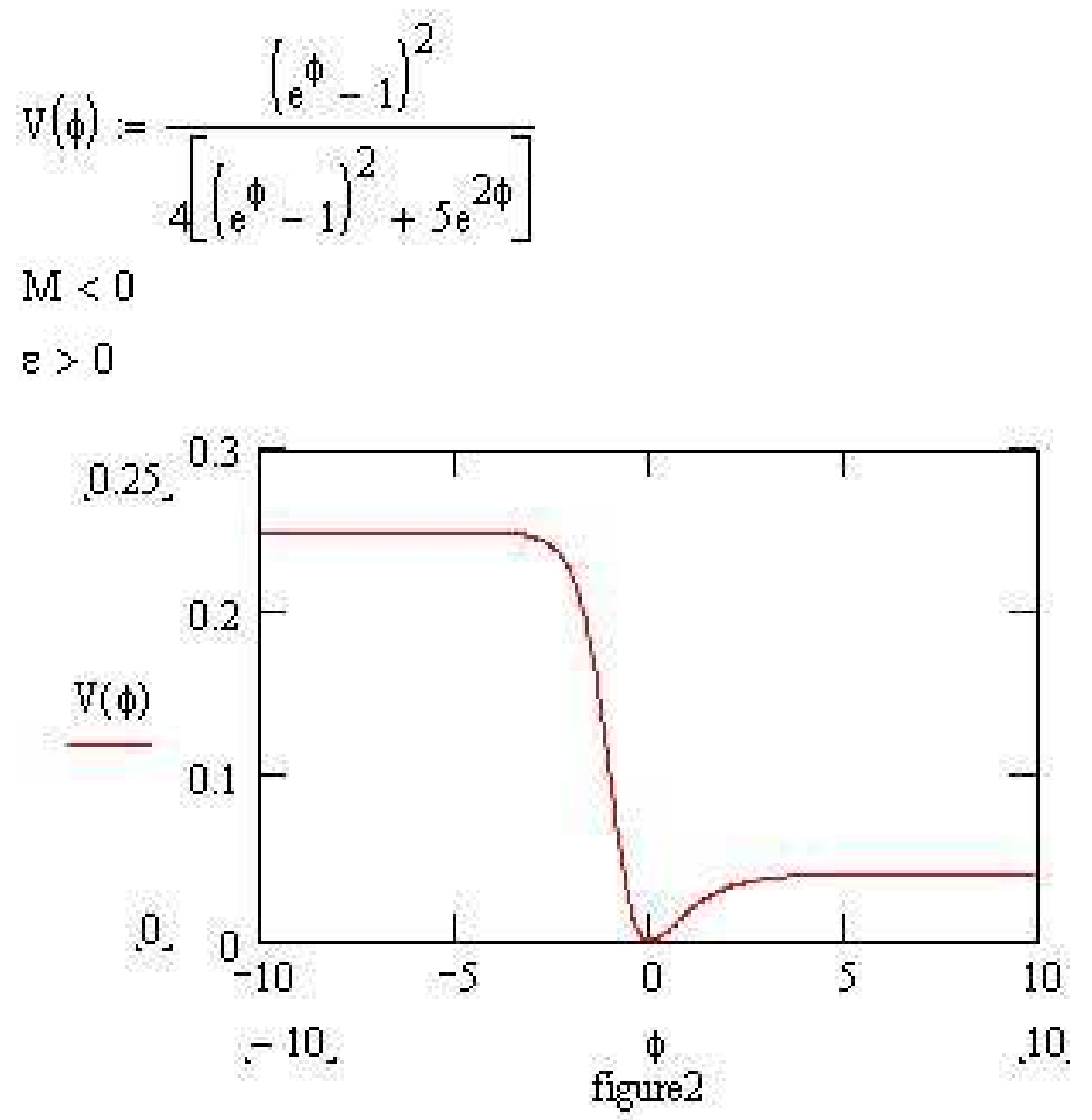




$$
V(\phi):=\frac{\left(e^{\phi}+1\right)^{2}}{4\left[\left(e^{\phi}+1\right)^{2}+5 e^{2 \phi}\right]}
$$

$M>0$.

$\varepsilon>0$

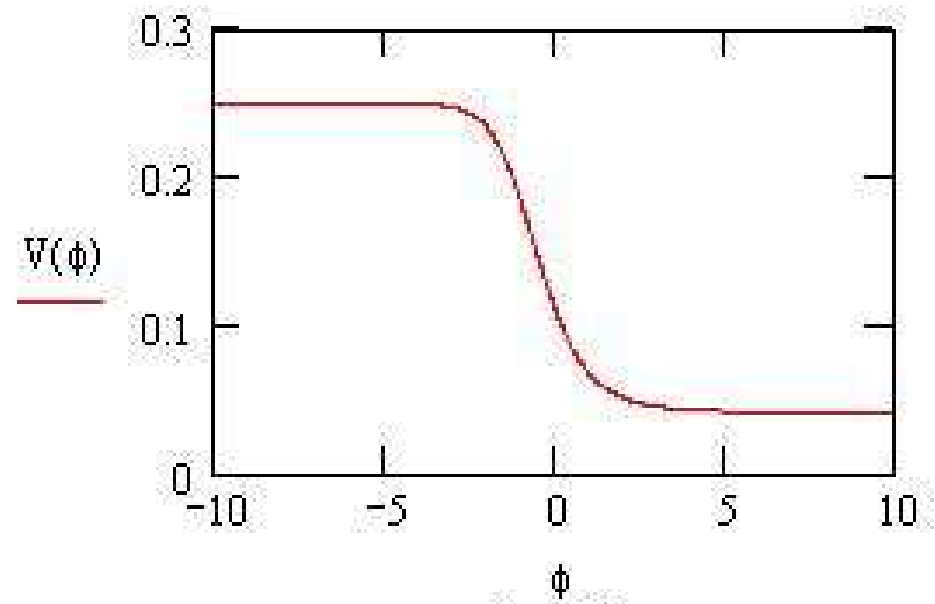

figure 3 